\title{
Evaluation of linezolid and vancomycin in the treatment of methicillin-resistant Staphylococcus aureus (MRSA) in Cystic Fibrosis patients
}

\author{
Celeste R. Caulder, PharmD ${ }^{1}$; P. Brandon Bookstaver, PharmD ${ }^{1}$; \\ David E. Brown III, MD $^{2}$; Daniel Brown, MD $^{2}$ \\ ${ }^{1}$ South Carolina College of Pharmacy - USC Campus, Columbia, SC \\ ${ }^{2}$ University of South Carolina School of Medicine, Division of Pediatric Pulmonology, Columbia, SC \\ *Corresponding author E-mail: caulderc@sccp.sc.edu
}

\begin{abstract}
Background: Cystic fibrosis (CF) patients often have pulmonary colonization with multiple organisms, including methicillin-resistant Staphylococcus aureus (MRSA), resulting in frequent hospitalizations and decreased quality of life. The pupose of this study was to evaluate the effectiveness of linezolid (LZD) and vancomycin (VAN) in the treatment of MRSA in the CF population.

Methods: A prospective, pilot study was conducted in CF patients with pulmonary exacerbation and sputum culture positive for MRSA randomized to receive intravenous LZD or VAN. The primary endpoint was microbiological eradication of MRSA and secondary endpoints included Kanga Clinical Score and change in quality of life questionaire (CF QOL) score. Patients were followed for 6 months post treatment. The ability to conduct a comprehensive outcome protocol in this population at a children's hospital was also evaluated.

Results: Eight subjects were randomized to receive LZD $(n=5)$ or VAN $(n=3)$, with average duration of therapy of 19.8 and 21 days, respectively. The primary endpoint of microbiological eradication at end of treatment (EOT) was achieved in 3 patients treated with LZD and 2 with VAN. Kanga Clinical Scores calculated at baseline and EOT showed a significant decrease in all subjects from a median of 26/50 to 16/50. Six patients or caregivers completed the CF QOL questionnaire. Five patients ( 2 treated with LZD, 3 with VAN) had improvements in CFQOL scores at EOT. No clinically significant adverse events were noted.

Conclusions: VAN and LZD were clinically effective and well tolerated in the treatment of MRSA in our CF population. Eradication of MRSA was less likely in patients with long-standing infection. Comprehensive study protocols such as this may be utilized in a large, randomized controlled trial to determine standardized treatment for newly acquired MRSA in CF patients.
\end{abstract}

Keywords: cystic fibrosis, vancomycin, linezolid, methicillin-resistant Staphylococcus aureus

\section{Introduction}

Staphylococcus aureus is an important pathogen in pulmonary infections in cystic fibrosis (CF) patients.[1-6] Vancomycin (VAN) has become a primary treatment option due to the increased prevalence of methicillin-resistant Staphylococcus aureus (MRSA) isolates; [1-4] however, with the emergence of S. aureus strains with increased VAN minimum inhibitory concentrations (MIC), decreased susceptibility, as well as clinical failures and potential nephrotoxicity, alternative regimens including trimethoprim-sulfamethoxazole, linezolid (LZD), and rifampin have been investigated.[2, 7-9, 10] Although there are a lack of data on the benefits of treating MRSA in CF patients, it is known that MRSA colonization may lead to increased systemic antibiotic use and potential lengthy hospitalizations that are costly to the healthcare system.[11,12] The purpose of this case series study was to evaluate a comprehensive protocol of clinical, microbiological, and radiological outcomes in hospitalized CF patients receiving either LZD or VAN for treatment of MRSA. 


\section{Materials and methods}

An Institutional Review Board approved case series study was conducted at a tertiary care children's hospital in South Carolina. Patients with a confirmed diagnosis of CF admitted for a pulmonary exacerbation were screened for study inclusion. Informed consent was obtained from patients and or parents/guardians prior to study enrollment. Subjects were included if they had a respiratory culture positive for MRSA at the time of admission. Patients with AST and/or ALT > 3 times upper limit of normal, concurrently receiving monoamine oxidase inhibitors, currently on transplant list, hemodialysis dependent chronic kidney disease, and known hypersensitivity to LZD or VAN were excluded.

Subjects received either one of two treatment arms: VAN $80 \mathrm{mg} / \mathrm{kg} /$ day (max initial dose 2 grams $/$ day) or LZD $10 \mathrm{mg} / \mathrm{kg}$ every 8-12 hours (max dose 600mg every 8 hours). Doses were adjusted by study investigators based on therapeutic drug monitoring and renal function. Subjects were hospitalized to receive a target minimum of 2 weeks of study medication. Any changes in therapy due to clinical deterioration or extension of therapy were at the discretion of the primary physician.

Baseline admission data were recorded to include the following: patient demographics; concurrent medications; microbiologic data in past 6 months to include previous cultures positive for MRSA and corresponding treatments; antibiotic exposure in last 90 days; and nutritional status. If possible, spirometry to include FEV 1 , FVC, and FEF25-75 was obtained.[13, 14] Spirometry was performed at the time of admission; at the end of weeks 1 and 2 of therapy; and 4 weeks post antibiotic therapy. End of week 3 spirometry was assessed in patients receiving therapy for greater than 2 weeks. Laboratory data including complete metabolic panel, liver function tests, C-reactive protein (CRP), and white blood cell count (WBC) were obtained at admission, at the end of weeks 1 and 2 of therapy, and 4 weeks post-antibiotic therapy. Radiographic imaging was performed at study entry and end of therapy and read by two pediatric radiologists blinded to treatment arms and scored using Brasfield scoring system.

Oropharyngeal and/or sputum cultures were obtained at admission, end of therapy (EOT), and 4 weeks post-antibiotic therapy. Culture and susceptibility data including specific MIC of study drugs were performed by the Clinical Microbiology Lab at the institution. The Kanga Clinical Score ${ }^{15}$ was calculated by study physician in all patients at admission and conclusion of study drug therapy. A Cystic Fibrosis Quality of Life (CFQOL) questionnaire [16] was given to all patients and parent/guardians at admission and conclusion of study drug therapy.

Descriptive analysis was performed to assess microbiological outcomes; spirometric measures of FVC, FEV1, FEF2575, clinical scoring system [15], CFQOL questionnaire score [17], length of hospital stay, and time until next positive culture for MRSA. For spirometry, patients were considered to be at baseline if the FVC, FEV1 and/or FEF25-75 were within $5 \%$ of non-exacerbation clinic visit values.

\section{Results}

Between June 2008 and April 2009, a total of 8 subjects received LZD ( $n=5)$ or VAN ( $n=3$ ). The median age was 8.5 years and 6 subjects were female (75\%). Subjects had a median length of stay (LOS) of 11 days. All 3 subjects who received VAN had a previous history of MRSA compared to 2 of 5 subjects who received LZD. Admission microbiological data showed 5 patients had polymicrobial infections. Two patients receiving VAN and 1 patient receiving LZD had only MRSA identified in respiratory culture. No patients had evidence of disseminated infection. No patients had a known exposure to VAN compared to 3 with a history of LZD in the previous 6 months. Minimum inhibitory concentration (MIC) obtained by E-test at admission showed a VAN and LZD MIC $90=2 \mathrm{mcg} / \mathrm{mL}$ and a VAN $\mathrm{MIC}_{50}=1.5 \mathrm{mcg} / \mathrm{mL}$ and $\mathrm{LZD} \mathrm{MIC} \mathrm{M}_{50}=2 \mathrm{mcg} / \mathrm{mL}$.

Table 1 below summarizes the demographic information and the clinical, laboratory and radiologic outcomes of the 8 study subjects. The average duration of therapy was 19.8 and 21 days in the VAN and LZD treated subjects respectively.

Six of 8 subjects had an elevated CRP at admission, resulting in an average $60 \%$ reduction in 5 subjects following 1 week of therapy; the greatest seen in 2 subjects in the LZD arm (subjects 4 and 6). Elevated WBC counts at admission had resolved in all patients by week 2 of therapy. The endpoint of change in spirometry at EOT was evaluable in 6 subjects. Two subjects in the LZD arm (subjects 4 and 6) experienced significant increases in FEV 1 by week 2. Admission $\mathrm{FEV}_{1}$ measurements in the VAN treated subjects were at or near baseline values. Similar results were seen for FVC, as 2 subjects in the LZD arm with significantly decreased FVC on admission had resulting increases by week 2. Kanga scores calculated at baseline and the EOT showed a significant decrease in all subjects from a median of $26 / 50$ to $16 / 50$. Chest $x$-rays at admission and at the EOT showed a small change in Brasfield scores from a median of 8.5 to 6.5. Six patients or caregivers completed the CFQOL questionnaire. Five of 6 patients had improvements in CFQOL scores at the EOT compared to admission.

The endpoint of microbiological eradication was achieved in 5 patients at the end of treatment (EOT). Two subjects who received LZD and 1 who received VAN had positive respiratory cultures for MRSA at EOT. Four weeks post 
therapy, 1 additional subject (total of 4) had positive respiratory cultures for MRSA. One subject in the VAN arm remained negative for 10-weeks post therapy. Three patients in the LZD arm, all without a prior history of MRSA, remained negative for 14, 15 and 68 weeks respectively. Of the 4 patients with evaluable E-test data at follow-up, MICs were increased in 3 of the subjects for their respective treatments.

No clinically significant adverse events including hematologic, renal or hepatic abnormalities were noted during the four weeks of study evaluation.

\section{Discussion}

The purpose of this case series study was to evaluate the effectiveness of VAN and LZD in the treatment of MRSA pulmonary infection in CF patients. In our small sample size, both regimens were tolerated well, with no clinically important, treatment-related adverse effects. Furthermore, both treatment regimens resulted in clinical improvement as manifested by changes in Kanga scores at the end of treatment. Though our study had a small sample size, it was likely that in our chosen patient population, improvement was likely regardless of the therapy used. All of the patients in this study were hospitalized because of pulmonary exacerbation of CF. In addition to antibiotics, the patients were treated aggressively with chest therapy, bronchodilators, and nutritional support-- all of which would augment antimicrobial therapy in the resolution of cough, increase in pulmonary function, and improved scores on CFQOL surveys.

In our study, 5 of 8 patients had eradication of MRSA at the end of treatment; 2 were treated with VAN, and 3 with LZD. Examination of the pre-study respiratory cultures shows that in the 3 patients in whom MRSA was eradicated by LZD, MRSA had been cultured for the first time. In all of the other patients, MRSA had been cultured previously. This long-standing infection presumably made eradication of MRSA much less likely, regardless of the treatment regimen. Of the 2 patients treated with VAN who experienced eradication of MRSA, one subsequently grew MRSA on a respiratory culture 4 weeks after treatment, which may indicate the MRSA was not completely eradicated (though reinfection is also a possibility). Again, a small study size hampers analysis, but our results do raise some interesting questions. First, it is possible, though likely more difficult, to eradicate MRSA in CF patients even though they may have had long-standing infection. Immediate treatment of new MRSA isolates in CF patients may be preferable, as has become the standard of care in newly acquired Pseudomonas aeruginosa infections.[18] Our study looked at patients experiencing pulmonary exacerbations, but aggressive therapy directed at newly colonized patients with MRSA who are otherwise asymptomatic may be even more successful. Furthermore, our patients were not started on additional protocols for the eradication of MRSA, which include topical treatment for nasal carriage of the organism, antimicrobial skin cleansers, and treatment of other family members in the home environment. The addition of these measures might make attempts at eradication of MRSA more successful and for a longer period of time.

Though VAN has long been the preferred antibiotic therapy for MRSA, decreased sensitivity to VAN in S. aureus is on the rise.[7] Furthermore, VAN requires intravenous administration, careful monitoring of drug levels, and has a risk of nephrotoxicity, even at appropriate serum concentrations. Penetration of the drug into lung tissue is also only a fraction of the serum concentration.[19] By comparison, LZD can be administered both orally and intravenously with equal bioavailability, requires no adjustment in renal or hepatic disease, and no therapeutic monitoring.[20,21] Perhaps most importantly, penetration into the epithelial lining fluid of the lung (the site of pulmonary infection in CF patients) is documented at $100 \%$ of serum concentrations.[21,22] Severe symptoms associated with serotonin toxicity have been shown in patients receiving linezolid and selected serotonin reuptake inhibitors.[23] There has only been one documented case of serotonin toxicity associated with concomitant linezolid use in CF patients. [24] No patients received serotonergic drugs in this study. Thrombocytopenia is common in patients taking linezolid. The majority of these adverse events develop after prolonged administration ( $>2$ weeks) or simultaneous administration with other drugs or food and subside after discontinuation of linezolid. Peripheral or optic neuropathy, another possible adverse effect, is associated with an even longer duration of linezolid treatment (3-6 months).[23] No LZD-related adverse events were noted in this case series.

\section{Conclusion}

Our data suggests that LZD is effective in eradicating MRSA in CF patients, and is well tolerated. Though our case series study was not designed to demonstrate differences in LZD versus VAN, LZD is no doubt more versatile and potentially safer. Meanwhile, the incidence of MRSA infection in CF patients and the population in general continues to rise.[25] Larger multicenter trials using LZD in CF patients with comprehensive microbiological, clinical and quality outcomes should be pursued so that a standardized treatment regimen for newly acquired MRSA, as well as patients with long standing MRSA, can be developed. Further investigation on the impact of other orally available antibiotics (trimethoprim/sulfamethoxazole, rifampin) and family/household directed anti-infective protocols is warranted. 


\section{References}

[1] Conway SP, Brownlee KG, Denton M, Peckham DG. Antibiotic treatment of multidrug-resistant organisms in cystic fibrosis. Am J Respir Med. 2003;2(4):321-332.

[2] Ferrin M, Zuckerman JB, Meagher A, Blumberg EA. Successful treatment of methicillin-resistant Staphylococcus aureus pulmonary infection with linezolid in a patient with cystic fibrosis. Pediatr Pulmonol. 2002;33:221-223.

[3] Thomas SR, Gyi KM, Gaya H et al. Methicillin-resistant Staphylococcus aureus: impact at a national cystic fibrosis centre. J Hosp Infect. 1998;40:203-209.

[4] Boxerbaum B, Jacobs MR, Chechner RL. Prevalence and significance of methicillin-resistant Staphyloccus aureus in patients with cystic fibrosis. Pediatr Pulmonol. 1988;4:159-163.

[5] Givney R, Vcikery A, Holliday A, et al. Methicillin-resistant Staphylococcus aureus in a cystic fibrosis unit. J Hosp Infect. 1977;35:27-36.

[6] Miall LS, McGinley NT, Brownlee KG, et al. Methicillin resistant Staphylococcus aureus (MRSA) infection in cystic fibrosis. Arch Dis Child. 2001;84:160-162.

[7] Smith TL, Pearson ML, Wilcox KR, et al. Emergence of vancomycin resistance in Staphylococcus aureus. N Engl J Med. 1999;340:493501.

[8] Drew R. Emerging options for treatment of invasive, multidrug-resistant Staphylococcus aureus infections. Pharmacother. 2007;27:227-249.

[9] Garske L, Kidd R, Gan R, et al. Rifampicin and sodium fusidate reduces the frequency of methicillin-resistant Staphylococcus aureus (MRSA) isolation in adults with cystic fibrosis and chronic MRSA infection. J Hosp Infect. 2004;56:208-214.

[10] Serisier DJ, Jones G, Carroll M. Eradication of pulmonary methicillin-resistant Staphylococcus aureus (MRSA) in cystic fibrosis with linezolid (correspondence). J Cystic Fibrosis. 2004;3:61.

[11] Solis A, Brown D, Hughes J, et al. Methicillin-Resistant Staphylococcus aureus in Children with Cystic Fibrosis: An Eradication Protocol. Pediatr Pulmonol. 2003;36:189-195.

[12] Ren C, Morgan W, Konstan M et al. Presence of Methicillin Resistant Staphylococcus aureus in Respiratory Cultures from Cystic Fibrosis Patients is Associated with Lower Lung Function. Pediatr Pulmonol. 2007;42:513-518.

[13] Beydon N, Davis SD, Lombardi E, et al. An Official American Thoracic/European Respiratory Society Statement: Pulmonary Function Testing in Preschool Children. Am J Respir Crit Care Med. 2007;175:1304-1345.

[14] Quittner AL, Buu A, Messer MA, et al. Development and Validation of the Cystic Fibrosis Questionnaire in the United States. Chest. 2005;128:2347-2354.

[15] Matouk E, Ghezzo RH, Gruber J, et al. Internal consistency reliability and predictive validity of a modified N. Huang clinical scoring system in adult cystic fibrosis patients. Eur Respir J. 1997;10:2004-2013.

[16] Miller MR, Crapo R, Hankinson J, et al. General Considerations for lung function testing. "ATS/ERS Task Force: Standardization of Lung function Testing.” Eur Respir J. 2005;26:153-161.

[17] Quittner AL, Buu A, Messer MA, et al. Development and Validation of the Cystic Fibrosis Questionnaire in the United States. Chest. 2005; $128: 2347-2354$

[18] Douglas TA, Brennan S, Gard S, et al. Acquisition and eradication of P. aeruginosa in young children with cystic fibrosis. Eur Respir J. 2009;33:305-11.

[19] Cruciani M, Gatti G, Lazzarini L, et al. Penetration of vancomycin into lung tissue. J Antimicrob Chemother. 1996;38:865-69.

[20] Slatter JG, Stalker DJ, Feenstra KL, et al. Pharmacokinetics, metabolism, and excretion of linezolid following an oral dose of linezolid to healthy human subjects. Drug Metab Dispos. 2001;29:1136-45.

[21] Ager S, Gould K. Clinical update on linezolid in the treatment of Gram-positive bacterial infections. Infect Drug Resist. 2012;5:87-102.

[22] Boselli E, Breilh D, Rimmele T, et al. Pharmacokinetics and intrapulmonary concentrations of linezolid administered to critically ill patients with ventilator associated pneumonia. Crit. Care Med. 2005;33:1529-33.

[23] Falagas M and Vardaka K. Benefit-risk assessment of linezolid for serious gram-positive bacterial infections. Drug Saf. 2008;31:753-68.

[24] Bergeron L, Boule M, Perreault S. Serotonin toxicity associated with concomitant use of linezolid. Ann Pharmacother. 2005;39:956-61.

[25] Cystic Fibrosis Foundation Patient Registry, 2011 Annual Data Report to the Center Directors. Bethesda, Maryland: Cystic Fibrosis Foundation, 2012. 
Table 1: Subject treatment outcomes $(n=8)$

\begin{tabular}{|c|c|c|c|c|c|c|c|c|}
\hline Characteristic & Subject 1 & Subject 2 & Subject 3 & Subject 4 & Subject 5 & Subject 6 & Subject 7 & Subject 8 \\
\hline Age, years & 20 & 16 & 16 & 10 & 7 & 7 & 5 months & 4 \\
\hline Gender & $\mathrm{F}$ & $\mathrm{F}$ & $\mathrm{F}$ & $\mathrm{F}$ & $\mathrm{M}$ & $\mathrm{F}$ & $\mathrm{M}$ & $\mathrm{F}$ \\
\hline Weight, kg & 55.5 & 49 & 43 & 27.6 & 19.8 & 14.7 & 6 & 15.2 \\
\hline $\begin{array}{l}\text { Study Treatment } \\
\text { Group (VAN or LZD) }\end{array}$ & LZD & VAN & VAN & LZD & VAN & LZD & LZD & LZD \\
\hline \multicolumn{9}{|l|}{ Microbiology Results } \\
\hline Admission & $\begin{array}{l}\text { MRSA, } \\
\text { PA }\end{array}$ & MRSA, PA & MRSA & $\begin{array}{l}\text { MRSA, } \\
\text { PA }\end{array}$ & MRSA & $\begin{array}{l}\text { MRSA, PA, } \\
\text { B. cepacia }\end{array}$ & $\begin{array}{c}\text { MRSA, } \\
\text { H. influenzae }\end{array}$ & MRSA \\
\hline EOT & $\begin{array}{l}\text { MRSA, } \\
\text { PA }\end{array}$ & No growth & $\begin{array}{c}\mathrm{PA}, \\
\text { C. albicans }\end{array}$ & $\begin{array}{l}\text { MRSA, } \\
\text { PA }\end{array}$ & $\begin{array}{l}\text { MRSA, } \\
\text { PA }\end{array}$ & B. cepacia & K. pneumoniae & $\begin{array}{l}\text { No } \\
\text { growth }\end{array}$ \\
\hline 4-Week Post Therapy & MRSA & No growth & MRSA, PA & $\begin{array}{l}\text { MRSA, } \\
\text { PA }\end{array}$ & $\begin{array}{l}\text { MRSA, } \\
\text { PA }\end{array}$ & $\begin{array}{l}\text { MSSA, } \\
\text { B. cepacia }\end{array}$ & No growth & $\begin{array}{l}\text { No } \\
\text { growth }\end{array}$ \\
\hline \multicolumn{9}{|l|}{ Laboratory Results } \\
\hline $\begin{array}{l}\text { FEV1/FVC/FEF 25-75 } \\
\text { (\%) (Admission) }\end{array}$ & $34 / 56 / 10$ & $76 / 88 / 49^{a}$ & $45 / 66 / 15$ & $45 / 54 / 25$ & $64 / 68 / 45$ & $31 / 42 / 13$ & N/A & N/A \\
\hline $\begin{array}{l}\text { FEV1/FVC/FEF } 25-75 \\
(\%)(E O T)\end{array}$ & $37 / 61 / 13$ & $80 / 95 / 45$ & $48 / 66 / 21$ & $67 / 74 / 48$ & $66 / 76 / 43$ & $57 / 63 / 36$ & N/A & N/A \\
\hline $\begin{array}{l}\text { CRP (mg/L) } \\
\text { (Admission) }\end{array}$ & 7.1 & 1.7 & 5.1 & 17.6 & 1.3 & 35.9 & 0.2 & 0.2 \\
\hline $\mathrm{CRP}(\mathrm{mg} / \mathrm{L})(E O T)$ & $3.1^{\mathrm{b}}$ & $<0.1$ & 0.7 & 5 & 9.5 & $2.1^{\mathrm{b}}$ & $<0.1$ & $<0.1$ \\
\hline $\begin{array}{l}\text { Brasfield Chest X-ray } \\
\text { Score (Admission) }\end{array}$ & 17 & 6 & 15 & 10 & 7 & 10 & 1 & 2 \\
\hline $\begin{array}{l}\text { Brasfield Chest X-ray } \\
\text { Score }(E O T)\end{array}$ & 15 & 3 & 14 & 10 & 4 & 9 & 1 & 4 \\
\hline \multicolumn{9}{|l|}{ Clinical Scoring Tools } \\
\hline $\begin{array}{l}\text { Kanga Score }^{\mathrm{c}} \\
\text { (Admission) }\end{array}$ & 31 & 26 & 28 & 34 & 23 & 26 & 16 & 24 \\
\hline Kanga Score $(E O T)$ & 18 & 13 & 19 & 19 & 19 & 14 & 11 & 11 \\
\hline $\mathrm{CFQOL}^{\mathrm{d}}$ (Admission) & 45.3 & 85.7 & 72.2 & 32.8 & 71.4 & $60.2^{\mathrm{e}}$ & N/A & N/A \\
\hline CFQOL (EOT) & 49.3 & 88.6 & 84.8 & 61.9 & 77.1 & 60.9 & N/A & N/A \\
\hline
\end{tabular}

VAN: vancomycin; LZD: linezolid; EOT: end of therapy; CRP: C-reactive protein; CFQOL: Cystic fibrosis quality of life; MRSA: methicillin-resistant Staphylococcus aureus; PA: Pseudomonas aeruginosa ${ }^{\mathrm{a}}$ Week 1 values used; ${ }^{\mathrm{b}} \mathrm{CRP}$ nadir was used from week 1 ; ${ }^{c}$ Maximum score of 50; ${ }^{\mathrm{d}}$ Patients $<6$ years of age, the parent/caregiver completed the quality of life questionnaire; ${ }^{\mathrm{C}}$ Parent score was used here as patient CFQOL at EOT was incomplete 